\title{
Pulp therapy in immature traumatized incisor using tricalcium silicate cement - a case report
}

\author{
Magdalena Gońda-Dominn ${ }^{1, A}$, Alicja Nowicka2, B, Karolina Węsierska ${ }^{1, C}$, Anna Jarząbek ${ }^{1, D}$ \\ Pomeranian Medical University in Szczecin, Department of Pediatric Dentistry, Powstańców Wlkp. 72/18, 70-111 Szczecin, Poland \\ 2 Pomeranian Medical University in Szczecin, Department of Conservative Dentistry and Endodontics, Powstańców Wlkp. 72/18, 70-111 Szczecin, Poland \\ A ORCID: 0000-0002-0479-8158; B ORCID: 0000-0002-0455-4209; C ORCID: 0000-0002-4575-9008; D ORCID: 0000-0001-7717-6141 \\ $\triangle$ gondzik@wp.pl
}

\begin{abstract}
Introduction: Traumatic injuries to immature permanent teeth are one of the most recurrent and urgent emergency treatment cases in pediatric dental practice. In many instances, the protocol for responding to this kind of emergency is not correct, and is not performed in a timely manner. Delays in seeking treatment following a complicated crown fracture in young individuals can result in irreversible pulpitis or pulp necrosis. It can leave a wide-open apex and brittle dentinal walls that are unfavorable to manage with conventional endodontic treatment (apexification).

Materials and methods: The present report describes the clinical case of a complicated crown fracture in young permanent central incisor, with 18 months of clinical and radiographic follow-up. The traumatized incisor was successfully treated using a coronal pulpotomy technique and tricalcium silicate cement - Biodentine $\mathrm{T}^{\mathrm{TM}}$
\end{abstract}

Results: The presented treatment approach provided elimination of the symptoms, an absence of pain episodes, and positive reactions to pulp sensitivity tests. The follow up radiological assessment revealed evidence of a dentin bridge formation and continuation of root maturation.

Conclusions: Coronal pulpotomies using tricalcium silicate cement are quick and easy to perform, and unlike endodontic treatment, preserve the tooth structure for the reattached tooth fragment. The restorative technique, the reattachment of fractured tooth fragment, and tooth restoration with a composite resin, represents an aesthetic, functional approach, and is an excellent and easy restorative option for clinicians and young impatient patients.

Keywords: Biodentine ${ }^{\mathrm{TM}}$; complicated crown fracture; immature permanent teeth; pulpotomy.

\section{INTRODUCTION}

Traumatic injuries to permanent teeth and their supporting tissues can occur at any age, but are rather more common in children and young adults. There is a particularly high prevalence of dental injuries in young male individuals between 9-10 years of age. The most frequent causes of trauma are from falls, traffic accidents, domestic violence, sports and fighting [1, 2]. Complicated crown fractures are from $2-13 \%$ of all dental injuries, with the most typical tooth involved, the maxillary central incisor [3]. Recently erupted teeth have short roots and their apices are wide, very often diverging. Dentine walls of immature teeth are thin, relatively weak and fragile. Loss of pulp vitality before the tooth has reached maturity may leave the tooth at risk of fracture. Endodontic treatment in such case can present a technical strain, which may diminish the long-term prognosis of the tooth $[2,3]$. The main goal after pulpal exposures in young permanent teeth is to preserve pulpal vitality, to allow continued physiological root formation. According to the articles of the European Society of Endodontology, when the dental pulp of a permanent tooth with incomplete root development is exposed, the therapeutic treatment should preserve the vitality of the remaining pulp tissue [4]. Partial pulpotomy or coronal pulpotomy is routinely a treatment of choice, but pulp capping can be also considered in case of minute pinpoint exposure $[2,5]$. After eliminating the injured pulpal tissue, it is recommended to use materials that protect the underlying healthy pulpal tissue, allowing and promoting the process of healing and repair [4, 5]. Delays in seeking treatment for complicated crown fractures of immature permanent teeth may lead to irreversible pulpitis that finally results in pulp necrosis [6]. These necrotic immature teeth have an interrupted root formation, leaving wide-open apexes and breakable dentinal walls that are unfavorable and inconvenient to manage with traditional endodontic treatment (apexification).

This case report describes the clinical case of a complicated crown fracture in a young permanent central incisor, with 18 months of clinical and radiographic follow-up. The traumatized incisor was successfully treated using coronal pulpotomy techniques, with the application of a tricalcium silicate cement - Biodentine $\mathrm{T}^{\mathrm{TM}}$ - and restoration of the tooth with a fragment reattachment procedure.

\section{CASE REPORT}

This case report presents the treatment approach in a 9-yearold male patient who had sustained a complicated crown fracture of the maxillary right central incisor (11), which had 
occurred due to a fall 2 days earlier. No foregoing treatment had been provided, and the crown fragment was stored in water. His main complaints were sensitivity to warmth and cold. Clinical and radiological examination revealed a crown fracture of tooth 11, involving the incisal 3 rd of the crown, with pulp exposure more than $2 \mathrm{~mm}$ (Fig. $1 \mathrm{a}$ and $1 \mathrm{~b}$ ). The response of the traumatized tooth (11) and the adjacent left central incisor (21) to cold testing was positive. No abnormal mobility of the injured incisor was observed, the periodontal tissues were healthy and the operative area was free of visible plaque. Treatment options were discussed with the patient and his parents, and a coronal pulpotomy with Biodentine ${ }^{\mathrm{TM}}$ was selected as the treatment of choice.

Local anaesthesia was administered (Citocartin 100, Molteni Dental, Italy), and the injured pulp tissue removed until healthy pulp tissue was reached, using a sterile diamond bur in a high-speed handpiece with copious water cooling. A cotton pellet soaked in $1.5 \%$ sodium hypochlorite was applied to the pulp wound with moderate pressure. After $5 \mathrm{~min}$, the bleeding had stopped, and Biodentine ${ }^{\mathrm{TM}}$ (Septodont, Saint-Maur-des-Fosses, France) was applied as a dressing material. A postoperative radiograph was taken (Fig. 1C). When the material had set, the enamel of the tooth remnant and the coronal fragment were etched with $35 \%$ phosphoric acid (UltraEtch, Ultradent Products, UT, USA) for 20 s, followed by extensive washing with air/water spray and subsequent drying with air. Then, a self-etching bonding system (G-Bond, GC Corporation, Tokyo, Japan) was applied over the fragments and UV light cured for $20 \mathrm{~s}$. The segment was reattached using a flowable composite and microhybrid composite resin (Gradia
Direct, Gradia flow, GC Corporation, Tokyo, Japan) - Figure 1d. Excess composite resin was removed before polymerization. All margins were cured for $40 \mathrm{~s}$ and then polished using diamond stones and a composite polishing kit (Shofu Co, Kyoto, Japan). Postoperative home instructions were given, and the patient was rescheduled for a 2 nd appointment. It was recommended that the patient rinse the mouth 2 ce a day with $0.1 \%$ chlorhexidine solution for a period of 7 days, carefully brush the teeth with a soft toothbrush and avoid incisal function as much as possible.

After a week, the patient returned and was asymptomatic. Follow-ups after 3, 6 and 18 months showed a fully functional, healthy tooth (Fig. 1e, 1f, 1g and 1h). The fractured tooth (11) and the adjacent incisor (21) showed a normal response to a cold test - there was an absence of pain, and there was no clinical or radiographic evidence of apical periodontitis or pathological resorption. Radiological assessments after 6 and 18 months revealed evidence of a dentin bridge formation and apical maturation (Fig. 1f and 1g).

\section{DISCUSSION}

Traumatic dental injuries to the anterior immature permanent teeth can not only inflict pain but also cause loss of function, loss of aesthetics and can easily influence psychological status, particularly in young individuals. Therefore, complicated crown fractures should be treated on an emergency basis to alleviate the symptoms and decrease the possibility of wound healing complications [6, 7]. Vital pulp therapies are
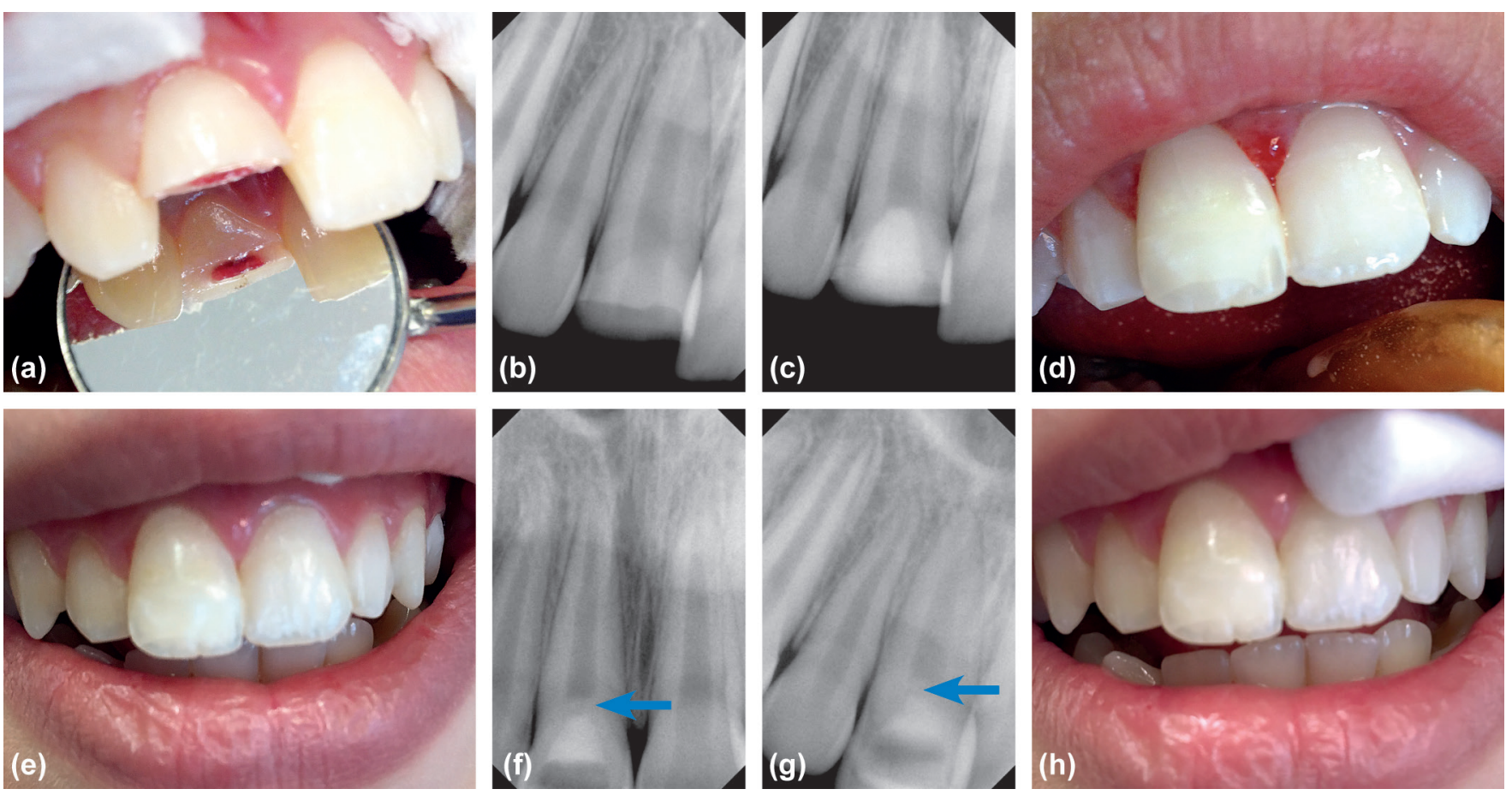

FIGURE 1. Clinical picture showing a complicated crown fracture in tooth 11 (a). Preoperative radiograph (b). Postoperative radiograph, after coronal pulpotomy using Biodentine ${ }^{\mathrm{TM}}$ (c). Clinical picture of the restored tooth $11(\mathrm{~d})$. Follow-up clinical picture of restored tooth 11 after 6 months (e). Follow-up radiograph at 6 months ( $\mathrm{f}$ ) and at 18 months $(\mathrm{g})$, showing dentine bridge formation and continued root maturation of traumatized tooth 11 . Follow-up clinical picture of the restored tooth 11 after 18 months (h) 
fully recommended in cases of complicated crown fractures to immature permanent teeth. Direct pulp capping and vital pulpotomy are the best choice, as they allow for continuing normal root growth $[1,2,4,5]$. The radicular pulp in immature permanent teeth has the extensive capacity to remain sound and maintain physiological functions. The general requirements for advantageous vital pulp therapy are: a normal pulp status prior to trauma, intact vascular supply to the pulp after trauma, exclusion of bacteria from the pulp excision zone during the healing period, the use of an appropriate vital techniques and biocompatible and bioactive material to encourage apexogenesis. The outcome of vital pulp therapy of the traumatized tooth may also depend on the type of tooth, the expanse and severity of the injury, the quality and timeliness of the initial care, the state of root formation, accompanying alveolar bone injury or associated injury to the periodontium, and the recall protocol $[1,2,5]$. After removing the injured pulpal tissue, it is recommended to use a material that protects the underlying sound pulpal tissues, promoting healing and repair process [7]. Studies have revealed that the principal discriminative factor between pulp capping materials is the speed and quality of dentin bridge creation. Calcium hydroxide is frequently identified in literature as the gold standard for vital pulp therapy and apexification. It allows for dentin bridge creation and continued root maturation $[1,2,8]$. However, calcium hydroxide has also been shown to form dentin containing tunnels favoring infection or necrosis of the pulp [8, 9]. An alternative biomaterial is mineral trioxide aggregate (MTA), which is generally used to cover exposed pulp in the anterior and posterior region, although it has a high potential for hard tissue staining $[10,11,12]$. Since it became commercially available in 2009, the tricalcium silicate cement Biodentine ${ }^{\mathrm{TM}}$ has been shown to have great bioactive properties for endodontic and restorative dentistry. Biodentine ${ }^{\mathrm{TM}}$ possess exclusive properties such as: biocompability, adequate adhesive ability, insolubility, dimensional stability and shorter setting time in contrast to MTA. Moreover, in vivo and in vitro studies have revealed that dentin bridge formation is more homogeneous and radicular dentin creation occurs faster with tricalcium silicate than with calcium-hydroxide based materials $[13,14$, $15,16]$. According to Możyńska et al. [11], Biodentine ${ }^{\mathrm{TM}}$ has the lowest hard tissue staining potential, which makes the material more suitable and efficient in cases of aesthetic restoration.

In the present study, we decided to use a coronal pulpotomy procedure with Biodentine ${ }^{\mathrm{TM}}$. Considerations were taken of the status of the pulp tissue, the expanse of the exposure, the intervening time between the unfortunate incident and the treatment, the patient's age and the maturity of the root. Although the most commonly used material for this technique is MTA or calcium hydroxide, in this case Biodentine ${ }^{\mathrm{TM}}$ was employed. Biodentine $^{\mathrm{TM}}$ reduces the number of required clinical sessions. It has previously been shown to have high success rates [16] and the lowest staining potential [11, 17].

Reattachment of the fractured tooth fragment is the most conservative and biologically sound method of restoring a fractured front tooth, allowing for reattachment of the dental fragment in 1 appointment. It is important that the tooth fragment is kept moist by storing it in a glass of water, to reduce the risk of future crown fracture. Flowable resin permits lap joining the fragments, and a minimum of restorative material is exposed on the surface along the fracture line. Coronal pulpotomies are quick and easy to perform, and unlike endodontic treatment, preserve the tooth structure for the reattached tooth fragment. Successful pulpotomy in immature permanent teeth can be demonstrated in clinical and radiological evidence. Clinical indicators of a successful pulpotomy are a positive normal response to pulp sensitivity tests and the absence of pain episodes. Radiological indicators of a successful pulpotomy in immature permanent tooth are continued root development, evidence of dentin bridge formation and an absence of radiological evidence of apical periodontitis and pathological resorption. Clinical and radiographic re-examinations were made 3, 6 and 18 months after the pulpotomy procedure: the patient showed a normal response to cold testing, the absence of pain episodes and had no clinical or radiographic evidence of pathological resorption or apical periodontitis. The radiological assessment after 6 months revealed evidence of dentin bridge formation and apical maturation.

\section{CONCLUSIONS}

Traumatic injuries, resulting in a loss of pulp vitality, can leave the tooth vulnerable to fracture, especially in immature permanent tooth, without complete closure of the apex. In such cases, endodontic treatment can present a technical hardship compromising the long-term prognosis of the tooth. The presented vital pulpotomy procedure with the use of tricalcium silicate cement, helped rescue the traumatized immature teeth by preserving pulp vitality and providing physiological continuation of root maturation. The restorative technique, including reattachment of the fractured tooth fragment and tooth restoration with a composite resin, represents an aesthetic and functional approach, and an excellent and easy restorative option for clinicians and young impatient patients.

\section{REFERENCES}

1. Andreasen JO, Bakland LK, Flores MT, Andreasen FM, Andersson L. Traumatic dental injuries: A manual. 3rd ed. Chichester: Wiley-Blackwell; 2011.

2. Welbury R, Whitworth, Duggal MS. Traumatic injuries to the teeth. In: Welbury R, Duggal MS, Hosey MT, editors. Paediatric dentistry. 4th ed. Oxford: Oxford University Press; 2012.

3. Cameron AC, Widmer RP, Abbott P, Heggie AA, Raphael S. Trauma management. In: Cameron AC, Widmer RP, editors. Handbook of pediatric dentistry. 4th ed. Sydney: Mosby Elsevier; 2013.

4. European Society of Endodontology. Quality guidelines for endodontic treatment: consensus report of the European Society of Endodontology. Int Endod J 2006;39(12):921-30.

5. American Academy of Pediatric Dentistry Council on Clinical Affairs. Guideline on management of acute dental trauma. Pediatr Dent 20082009;30(7 Suppl):175-83. 
6. Andreasen JO, Andreasen FM, Skeie A, Hjørting-Hansen E, Schwarz O. Effect of treatment delay upon pulp and periodontal healing of traumatic dental injuries - a review article. Dent Traumatol 2002;18(3):116-28.

7. Bimstein E, Rotstein I. Cvek pulpotomy - revisited. Dent Traumatol 2016;32(6):438-42.

8. Huang GTJ. Apexification: the beginning of its end. Int Endod J 2009;42(10):855-66.

9. Andreasen JO, Farik B, Munksgaard EC. Long-term calcium hydroxide as a root canal dressing may increase risk of root fracture. Dent Traumatol 2002;18(3):134-7.

10. Kang SH, Shin YS, Lee HS, Kim SO, Shin Y, Jung IY, et al. Color changes of teeth after treatment with various mineral trioxide aggregate-based materials: an ex vivo study. J Endod 2015;41(5):737-41.

11. Możyńska J, Metlerski M, Lipski M, Nowicka A. Tooth discoloration induced by different calcium silicate-based cements: A systemic review of in vitro studies. J Endod 2017;43(10):1593-601.

12. El-Meligy OAS, Avery DR. Comparison of mineral trioxide aggregate and calcium hydroxide as pulpotomy agents in young permanent teeth (apexogenesis). Pediatr Dent 2006;28(5):399-404.
13. Mohammadi Z, Dummer PMH. Properties and applications of calcium hydroxide in endodontics and dental traumatology. Int Endod J 2011;44(8):697-730.

14. Bakland LK, Andreasen JO. Will mineral trioxide aggregate replace calcium hydroxide in treating pulpal and periodontal healing complications subsequent to dental trauma? A review. Dent Traumatol 2012;28(1):25-32.

15. Nowicka A, Lipski M, Parafiniuk M, Sporniak-Tutak K, Lichota D, Kosierkiewicz A, et al. Response of human dental pulp capped with Biodentine and mineral trioxide aggregate. J Endod 2013;39(6):743-7.

16. Koubi G, Colon P, Franquin JC, Hartmann A, Richard G, Faure MO, et al. Clinical evaluation of the performance and safety of a new dentine substitute, Biodentine, in the restoration of posterior teeth - a prospective study. Clin Oral Investig 2013;17(1):243-9.

17. Shokouhinejad N, Nekoofar MH, Pirmoazen S, Shamshiri AR, Dummer $\mathrm{PMH}$. Evaluation and comparison of occurrence of tooth discoloration after the application of various calcium silicate-based cements: an ex vivo study. J Endod 2016;42(1):140-4. 